\title{
Innovative Thermal Management Concepts and Material Solutions for Future Space Vehicles
}

\author{
Burkard Esser* \\ DLR, German Aerospace Research Center, 51147 Cologne, Germany \\ Jorge Barcena \pm \\ Tecnalia, 20009 San Sebastian, Spain \\ Markus Kuhn \\ DLR, German Aerospace Research Center, 70569 Stuttgart, Germany \\ Altug Okan $\stackrel{\S}{-}$ \\ Middle East Technical University, 06800 Balgat, Ankara, Turkey \\ Lauren Haynes $\mathbb{1}$ \\ Fluid Gravity Engineering, Ltd., Emsworth, England PO10 7DX, United Kingdom \\ Sandro Gianella** \\ EngiCer, S.A., 6828 Balerna, Switzerland \\ Alberto Ortonati \\ Scuola Universitaria Professionale della Svizzera Italiana, 6928 Manno, Switzerland \\ Volker Liedtke \\ Aerospace and Advanced Composites, GmbH, 2700 Wiener Neustadt, Austria \\ Daniele Francesconi $\underline{\S \S}$ \\ Thales Alenia Space Italia Spa, 10146 Torino, Italy \\ and \\ Hideyuki TannoI \\ Japan Aerospace Exploration Agency, Kakuda Miyagi 981-1525, Japan
}

\section{DOI: $\underline{10.2514 / 1 . A 33501}$}

\begin{abstract}
When entering a planetary atmosphere, space vehicles are exposed to extreme thermal loads. To protect the vehicle's interior, a thermal protection system is required. Future aerospace transportation demands solutions that exceed the performance of current systems and up-to-date material limits. Therefore, new and disruptive solutions must be envisaged to meet those extreme conditions. In the search of new solutions for sharp leading edges of future hypersonic reentry or transport vehicles, the THOR project, composed of eight European organizations (industries, research centers, and universities) and one Japanese Agency (Japan Aerospace Exploration Agency), is actively working on definition, design, implementation, and simulation of new passive and active thermal management solutions and their verification in relevant environments (high-enthalpy facilities). This paper provides an overview of the recent developments on the four concepts that are targeted in the project, applying different physical methodologies: 1) passive cooling using highly conductive carbon-based fibers, 2) passive cooling with intensive internal radiative exchange, 3 ) active cooling based on convection heat transfer using a ceramic sandwich/thermal protection system with ceramic foams/lattices, and 4) active transpiration cooling of external surfaces. Details on these thermal management concepts, requirements from end users, and test configurations, as well as results from experimental and numerical verification, are given.
\end{abstract}

Received 3 November 2015; revision received 8 April 2016; accepted for publication 13 April 2016; published online XX epubMonth XXXX. Copyright $\odot 2016$ by Fundación Tecnalia Research and Innovation. Published by the American Institute of Aeronautics and Astronautics, Inc., with permission. Copies of this paper may be made for personal and internal use, on condition that the copier pay the per-copy fee to the Copyright Clearance Center (CCC). All requests for copying and permission to reprint should be submitted to CCC at www.copyright.com; employ the ISSN 0022-4650 (print) or 1533-6794 (online) to initiate your request.

*Project Manager, Institute of Aerodynamics and Flow Technology.

†Project Manager, Aerospace Area, Industry and Transport Division, Mikeletegi Pasealekua 2. Member AIAA.

${ }^{\ddagger}$ Project Engineer, Institute of Structures and Design.

\$Project Engineer, Tubitak Uzay Space Technology Research Institute.

"Project Engineer, Old Coach House, 1 West Street.

**Project Engineer, Viala Pereda 22.

${ }^{\dagger}$ Full Professor, Instituto CIM per la sostenibilità nell'innovazione, Galleria 2.

\#Senior Researcher, Viktor-Kaplan-Str. 2.

$\$_{\$}$ Head of Department, Strada Antica di Collegno 253.

ITProject Manager, Kakuda Space Center.

$\begin{aligned} & \text { Nomenclature } \\ h & =\text { enthalpy } \\ \dot{q} & =\text { heat flux } \\ R & =\text { nose radius } \\ u & =\text { velocity of fluid } \\ \rho & =\end{aligned}$

\section{Subscripts}

$\mathrm{cw}=$ cold wall

hw $=$ hot wall

ref $=$ reference or local value

$\infty=$ flight or freestream data

\section{Introduction}

$\mathbf{T}$ HERMAL protection systems (TPSs) are required to protect the internal structure and payloads of space vehicles entering a planetary atmosphere from outer space. There are several examples, such as manned orbiters like the space shuttle, probe missions, or planetary exploration missions (e.g., to Mars). For this purpose, it is 
necessary to protect the vehicle from all heat generated during reentry. This can be carried out by proper design as well as selection of appropriate high-temperature materials. There are many different possibilities, but the final selection depends on the vehicle's entry velocity, its shape and size, its entry trajectory, and the properties of the atmosphere.

The research project THOR funded within the European Union's FP7 program is targeting improvements of the thermal management for ceramic matrix composite (CMC)-based reusable thermal protection systems [1]. In the case of a passive TPS, state-of-the-art thermal management is based on local heating conditions, which are in a quite straightforward procedure used to determine the main parameters of thermal protection, resulting in the typical blunted geometries.

The classical local thermal management concept does not account for significant gradients of thermal loads along the surface. Such gradients, however, are expected to become significant for future space vehicles as well as for some concepts of hypersonic transport vehicles, which are currently being considered [2], such as Skylon [3], SpaceLiner [4], LAPCAT [5], ATTLAS II [6], and so on. Most of these vehicles are based on an improved aerodynamic performance, which requires a reduction of bluntness, in particular at the wings' leading edges. Accordingly, the regions with lowest radius of curvature and highest thermal loads get reduced in size and become immediately surrounded by areas with a substantially lower loading. The absolute values of the maximal heat fluxes, however, will increase significantly with a reduced radius of curvature. A typical method to assess the heat flux is the Detra-Kemp-Riddell relation [7], as shown in Eq. (1):

$$
\dot{q}=\dot{q}_{\mathrm{cw}}=\frac{a}{R^{0.5}}\left(\frac{\rho_{\infty}}{\rho_{\mathrm{ref}}}\right)^{0.5}\left(\frac{u_{\infty}}{u_{\mathrm{ref}}}\right)^{3.15}
$$

This relation is providing the so-called cold wall heat flux that is supposed to be a conservatively assessed maximal heat flux, because it is considering the wall at low temperatures. The heat flux is governed by three parameters. Two of them are trajectory data (i.e., freestream density $\rho_{\infty}$ and the vehicle's flight velocity $u_{\infty}$ ). The third parameter refers to geometry: It is the radius of curvature $R$. In this context, heat flux is directly linked to a design parameter. It is inversely proportional to the square root of $R$, and accordingly, the heat flux increases when reducing bluntness.

Finite wall temperatures (e.g., for a steady state) are being evaluated based on the cold wall values and by considering a correction factor that takes into account the enthalpy differences between cold and hot wall states, as shown in Eq. (2):

$$
\dot{q}_{\mathrm{hw}}=\dot{q}_{\mathrm{cw}}\left(\frac{h_{0}-h_{\mathrm{hw}}}{h_{0}-h_{\mathrm{cw}}}\right)
$$

For systems with considerable load gradients, the classical local thermal management must be considered a zeroth-order solution only. Therefore, the local concept is not being followed in THOR. It is being replaced by more global thermal balances that consider thermal loadings on the complete surface of a vehicle or one of its components.

In this sense, a set of mission scenarios and general requirements on potential evolutions of hypersonic applications (beyond 2020) have been specified by Thales Alenia Space, who are taking part in the project as a prospective end user. The stepwise approach has consisted of outlining the end-user needs, to bridge the current atmospheric reentry technologies toward the settlement of actually considered configurations (see Fig. 1).

The following steps are outlined as follows: 1) short-/mediumterm developments, a reference application deemed as evolution of the European state-of-the-art in Space Entry Vehicles technology and its current industrial capabilities; 2) medium-/long-term development, evolutions in space entry vehicle technologies, altogether in parallel to current technological investigations of novel propulsion technologies; and 3) future applications, hypersonic vehicles (speed in the range of Mach greater than 5).

Based on the tradeoff of different missions it was concluded that a wing leading edge was defined as a baseline configuration to be studied in THOR, to satisfy the requirements for future vehicles, where reusability on winged bodies is demanded and the use of hot structures based on ceramic matrix composites is a must. Nevertheless, in case of vehicles with nearly sharp leading edges, it seems that passive thermal protection will exceed the current usage

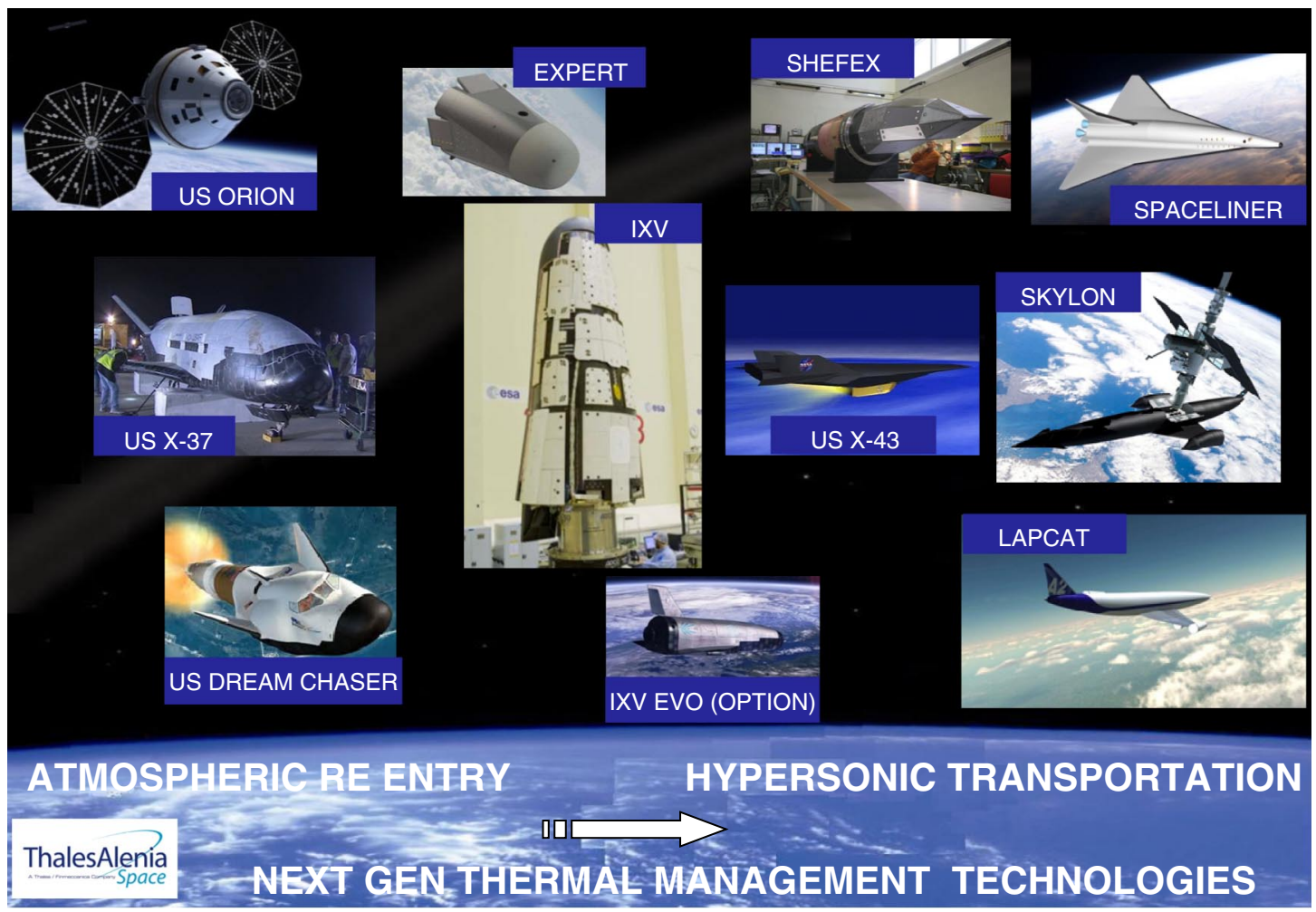

9 Fig. 1 Current considered configurations for atmospheric entry and hypersonic transport. 
Table 1 Summary of concepts

\begin{tabular}{ll}
\hline \hline $\begin{array}{l}\text { Type of } \\
\text { cooling }\end{array}$ & \multicolumn{1}{c}{ Description } \\
\hline Passive & $\begin{array}{l}\text { (1a) Passive cooling using highly conductive carbon-based } \\
\text { fibers } \\
\text { (1b) Passive cooling with intensive internal radiative exchange } \\
\text { Passive }\end{array}$ \\
Active & $\begin{array}{l}\text { (2a) Active cooling using a ceramic sandwich-TPS with } \\
\text { ceramic foam }\end{array}$ \\
Active & (2b) Active transpiration cooling of external surfaces \\
\hline \hline
\end{tabular}

limits of those materials at service conditions (i.e., heat fluxes up to $2 \mathrm{MW} / \mathrm{m}^{2}$, leading to a temperature limit of $2000 \mathrm{~K}$ ), even with a global thermal management. Therefore, thermal management technologies with active cooling are also considered and are going to be verified in THOR.

\section{Thermal Management Concepts}

For systems with considerable load gradients, the classical local thermal management must be considered a zeroth-order solution only. Therefore, the local concept is not being followed in the frame of THOR. It is being replaced by more global thermal balances that consider thermal loadings along the complete surface of a vehicle or one of its components. A leading-edge geometry was selected as the reference geometry for the project.

The global approach is supposed to provide more realistic thermal data, which allow for increasing the tolerable heat loads while maintaining the actual operating limit temperatures of CMC surface materials. In addition, appropriate physical and technological requirements are considered. These support the global thermal management approach with the goal of improving the performance of thermal protection systems by 1) equilibrium of thermal loading by transferring heat efficiently from highly loaded surface areas to less loaded regions, or 2) reduction of surface temperatures by means of active cooling.

The consideration of global thermal management concepts is encouraged by progress in base material development. New materials with tailored properties are available for being integrated into thermal protection systems as, for example, 1) pitch-based fibers with very high thermal conductivity that exceeds the conductivity of conventional fibers by at least one order of magnitude, as well as 2) SiC-based ceramic foams and lattices with minor damage tolerance but operational temperatures being very close to those of conventional dense CMC materials.

Experimentally, in the frame of this project, a significant potential has been observed and validated for the reduction of surface temperatures by the long-distance thermal-exchange capabilities of thermal radiation and transpiration cooling of external surfaces. These developments and results are investigated to quantify their specific potential with regard to the objectives (i.e., equilibration of thermal loads and reduction of surface temperatures). In total, four different concepts are being considered, grouped into passive cooling concepts and active cooling, as shown in Table $\underline{1}$.
Within each group, two separate thermal concepts are investigated. Each concept is going to be developed in detail, transferred and implemented into hardware, and validated experimentally in highenthalpy facilities and numerically by coupled simulations in parallel.

\section{A. Passive Cooling Concepts}

Efficient thermal equilibration is the principal objective for the thermal management concepts using passive cooling technologies. The thermal load balance shall be improved by transferring heat from highly loaded areas to regions with a lower thermal loading. Of course, the potential of passive cooling technologies is restricted when being compared with active cooling. Nevertheless, it is useful to identify and quantify the potential, because passive cooling does not require integration of any additional auxiliary systems for space application. The two considered passive cooling concepts are 1) concept $1 \mathrm{a}$, innovative composite materials with integrated highly conducting fibers; and 2) concept $1 \mathrm{~b}$, TPS structures with intensive radiative heat exchange.

In concept $1 \mathrm{a}$, the heat conduction capability of the TPS skin is being improved by integrating highly conductive fibers into the ceramic matrix. Conventionally, fibers with a conductivity of $10-20 \mathrm{~W} / \mathrm{mK}$ are being used. There are, however, pitch-based carbon fibers with a reported thermal conductivity of up to $600 \mathrm{~W} / \mathrm{mK}$. An increase of the fibers' conductivity by at least one order of magnitude is expected to be realistic, based both on a block circuit (BC) and finite element (FE) model. The influence on the effective thermal conductivity of the composite was analyzed by Reimer et al. [8]. The results shown in Fig. 2 were obtained under the assumption that the fibers' conductivity does not change at elevated temperatures. The data confirm the expectation that the thermal conductivity of a CMC material can considerably be improved when using fibers with a high conductivity.

Concept $1 \mathrm{~b}$ is approaching thermal equilibration by heat exchange below the TPS skin. Advantage is taken from the long-distance thermal-exchange capabilities of thermal radiation. According to the Stefan-Boltzmann relation, radiated heat strongly intensifies with temperature and therefore intrinsically supports thermal equilibration on a nonuniformly loaded curved structure. The potential of this mechanism for conventional CMC-based thermal protection had been demonstrated by thermal tests on a flat plate configuration [9].

\section{B. Active Cooling Concepts}

Active cooling is an attractive option, because it provides the potential of carrying away large amounts of heat or preventing heat loads to impinge. The method, however, adds complexity to the system, because it requires additional system components (e.g., coolant storage, supply and return pipes). Therefore, active cooling has not been considered for space vehicles in the past, and for the future, it is supposed that active cooling is implemented only if passive cooling concepts are not sufficient to keep a thermal protection system within its operational limits.

The two active cooling concepts that are considered in THOR are 1) concept $2 a$, a sandwich-TPS with ceramic foams; and 2) concept $2 b$, transpiration cooling. The two concepts reflect the two different

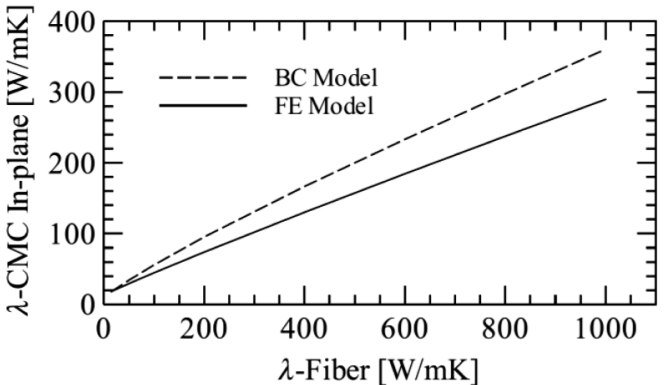

a) In fibre plane

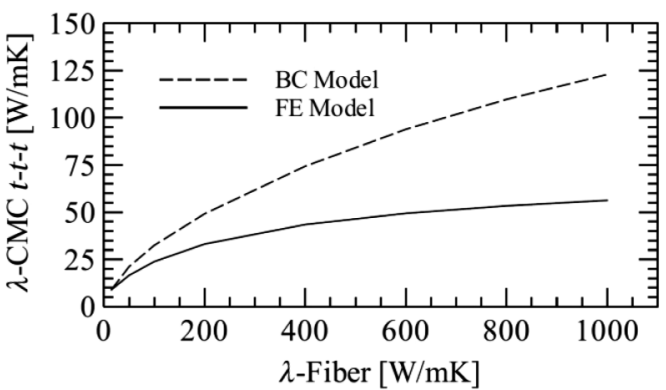

b) Orthogonal to fibre plane

Fig. 2 Actually considered configurations for atmospheric entry and hypersonic transport. 


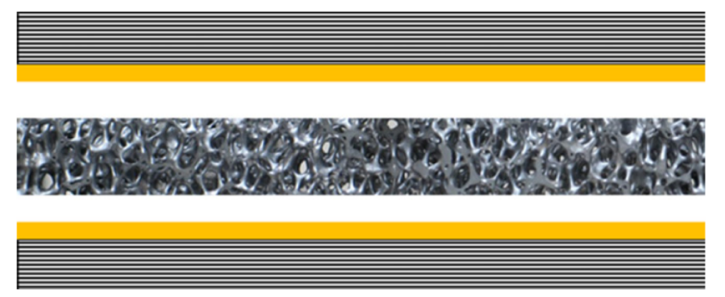

$\mathrm{CMC}$

Fig. 3 Sketch of ceramic sandwich.

technical approaches that are principally used to actively cool a highly loaded surface by leading the coolant either along the backside of the hot surface or pushing it through a porous segment immediately to the external surface.

In concept $2 \mathrm{a}$, a novel ceramic sandwich structure, as it was described in [10], is being considered. In its most general form, it is made of two ceramic skin layers and a silicon-carbide-based ceramic foam in between, as shown in Fig. 3. When applied in a TPS structure, one of the CMC layers would be identical to a vehicle's external CMC skin. The foam, which might be replaced by a lattice structure, is highly porous and therefore well suited to pass the coolant along the backside of the external skin to highly loaded regions, where it takes over the heat convectively, additionally reducing the surface temperature, taking advantage of the increased surface for heat exchange and of the high thermal conductivity of the SiC-based struts as well.

The cooling principle of concept $2 \mathrm{a}$ is based on convective cooling. Accordingly, there is no necessity to specifically demonstrate the physical validity of this thermal management approach. Nevertheless, there are technological challenges that are mainly related to finding the optimal morphology of the foam and identifying suitable joining techniques for the interface of foam and external CMC skin.

For transpiration cooling in concept $2 \mathrm{~b}$, the coolants are supposed to be fed through porous surface elements to the exterior. In this case, cooling is achieved not only for the porous element itself, but also for regions downstream of transpired surface parts. Transpiration cooling has successfully been applied for CMC-based structures in various research projects (e.g., RESPACE [11] and FAST20XX [12]). Transpiration cooling does not require a closed loop for the coolant. Therefore, the auxiliary infrastructure is not as complex as required for an application of the ceramic sandwich in concept $2 \mathrm{a}$.

There is one major drawback of transpiration cooling, because the emanating coolant might considerably interfere with the boundary layer that is established on the external surface. Depending on the type of coolant and its flow rate, the coolant is able to trigger boundary-layer separation or initiate boundary-layer transition. At flight relevant Mach and Reynolds numbers, both phenomena are potentially associated with highly intensified heat loads in the wake of the porous segment, leading to a considerable reduction or even reversion of global thermal performance.

\section{Test Configurations}

As a result of the general assessment of future requirements, a leading-edge geometry was selected for experimental and numerical verification in the frame of the THOR project. Two different geometries are being considered. The "baseline geometry" includes a leading edge with a nose radius of $20 \mathrm{~mm}$. A smaller nose radius of $10 \mathrm{~mm}$ was chosen for the second option. The principal setup for experimental verification is identical for both geometries. As sketched in Fig. 4a, the assemblies are mounted on a water-cooled base plate (1). This base plate is an artifact incorporated to the test article, to keep its down structure cold (to protect gas inlets, thermocouple connection, and other subsystems safe). Other common elements are the TPS structure (2), a copper connector (3), and CMC side (4) and rear (5) walls.

A conventional passively cooled TPS structure is considered as reference concept " 0 ". It is sketched in Fig. 4 b. In this case, the TPS structure (2) consists of the external skin only, prepared with conventional CMC material. In addition to the components illustrated in Fig. 4a, a block of insulation material (6) fills the space between $\mathrm{CMC}$ skin and water-cooled base plate to minimize thermal losses to the interior. The sketch in Fig. $4 \mathrm{~b}$ is describing the test configuration of concept $1 \mathrm{a}$ as well. The $\mathrm{CM} \overline{\mathrm{C}}$ skin of this configuration, however, is being prepared with integrated highly conductive fibers that are aligned parallel to the surface.

For concept $1 \mathrm{~b}$, internal radiation will be enabled by removing the insulation block used for concepts 0 and 1a, either partially or completely. The CMC skin is made of conventional standard CMC. In a fully passive version, the insulation is being removed in the curved part only, as sketched in Fig. 5. It allows radiative heat exchange for thermal equilibration in the nose region.

When removing the insulation block completely, which is not joined with the upper skin (just placed in tight contact), the internal radiation can further be intensified. Because the CMC skin can radiate to the water-cooled base plate, the concept is not a fully passive one anymore, but becomes semi-active. The semi-active version is described by the sketch in Fig. 4a. It is considered as a limiting case only (for demonstration purposes during the test campaign), because such a high visibility of actively cooled parts seems unrealistic for an application (as, e.g., a leading edge or a nosecap). By replacing the standard $\mathrm{CMC}$ with a $\mathrm{CMC}$ prepared with highly conductive fibers, concepts $1 \mathrm{a}$ and $1 \mathrm{~b}$ can be combined.

Concept $2 \mathrm{a}$ is aiming for active convective cooling of the CMC skin from the backside. This is achieved by integration of a ceramic $\mathrm{Si}$-SiC lattice as a central part of a sandwich that the coolant can be passed through. As shown in Fig. 6, the lattice (7) is covering the backside of the external CMC skin in the nose region. The coolant is supplied from outside through a leadthrough in the base plate, which is also used for instrumentation. It also extends downstream for some distance along the top surface, providing additional potential for

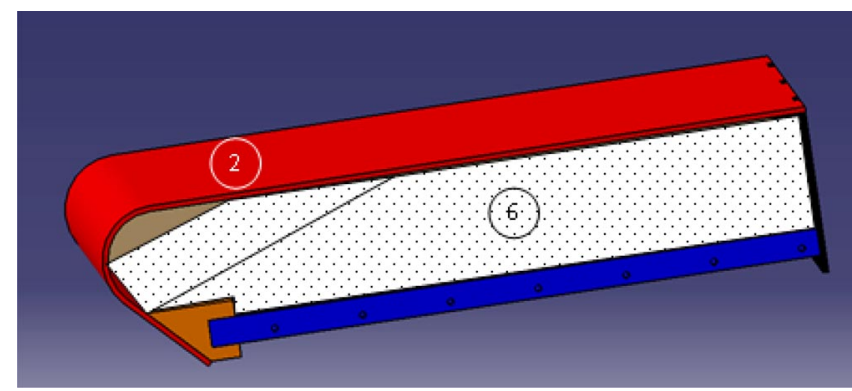

Fig. 5 Test configuration for concept $1 \mathrm{~b}$ (fully passive version).

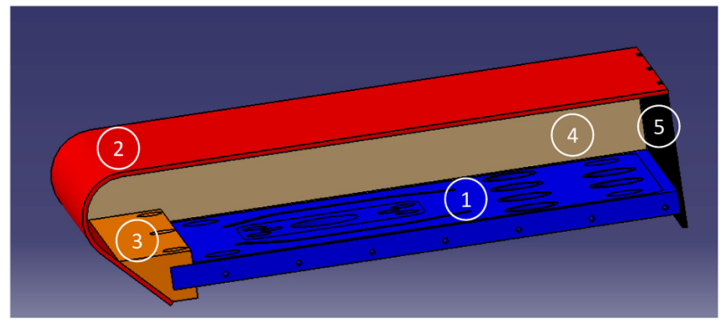

a) Baseline geometry $(R=20 \mathrm{~mm})$

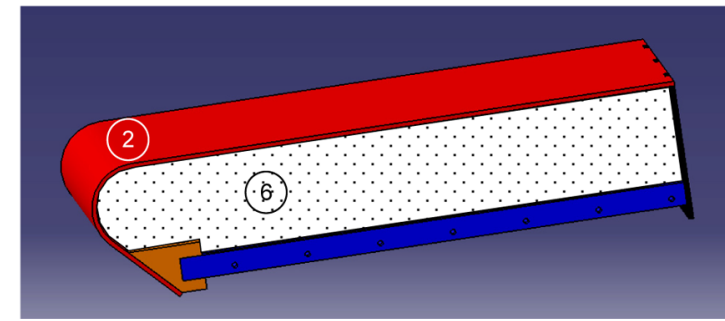

b) Reference concept " 0 "

Fig. 4 Actually considered configurations for atmospheric entry and hypersonic transport. 


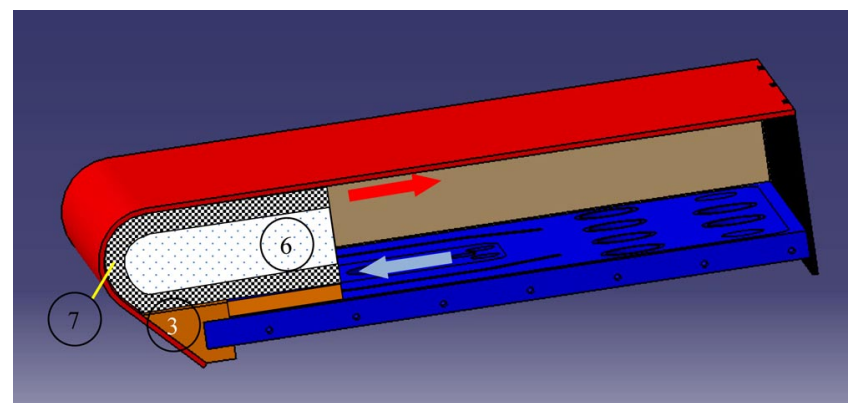

Fig. 6 Test configuration for concept $2 a$.

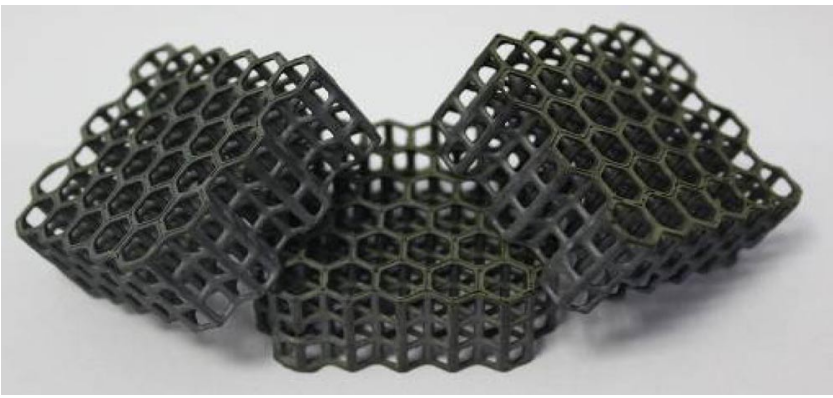

14 Fig. 7 Si-SiC lattice for concept $2 \mathrm{a}$.

thermal equilibration. Along this additional length, the lattice will tightly be joined to the CMC skin. This CMC skin is made of $\mathrm{SiC} /$

$12 \mathrm{SiC}$, because this ceramic composite will have a coefficient of thermal expansion closer to the $\mathrm{Si}$-SiC lattice, reducing the thermal mismatch and therefore the probability of failure due to thermal stresses.

A block of insulation material (6) is used to form a channel for the coolant flow along the CMC skin. The upper leg of the copper connector (3) will be adapted to the length of the foam to keep the external geometry of the foam simple. In a basic preparatory step, optimal manufacturing process and lattice morphology were 3 identified by the project team members Erbicol and Scuola Universitaria Professionale della Svizzera Italiana. The selected lattice morphology, which is shown in Fig. 7, was characterized concerning its fluid dynamic and mechanical properties.

To identify the optimal joining technique for the interface of lattice and external CMC skin, a tradeoff study was initiated. Starting from five initial processing routes, three were preselected to be further assessed, which include the use of: 1) high-temperature adhesives based on $\mathrm{SiC}$ (commercially available), 2) preceramic polymers (polysilazane), mixed with different fillers ( $\mathrm{Si}, \mathrm{SiC}, \mathrm{MAX}$ phases) to promote beneficial reaction and improve the stability and performance, and 3) similar to the previous, where phenolic resin is used instead of the preceramic polymers. Although the first method is based on a quite easy processing route (application and curing at $90^{\circ} \mathrm{C}$ ), the last two methods involve a rather complex route with several steps: curing at autoclave, pyrolysis, and final treatment at temperatures above $1400^{\circ} \mathrm{C}$ under controlled atmosphere conditions.

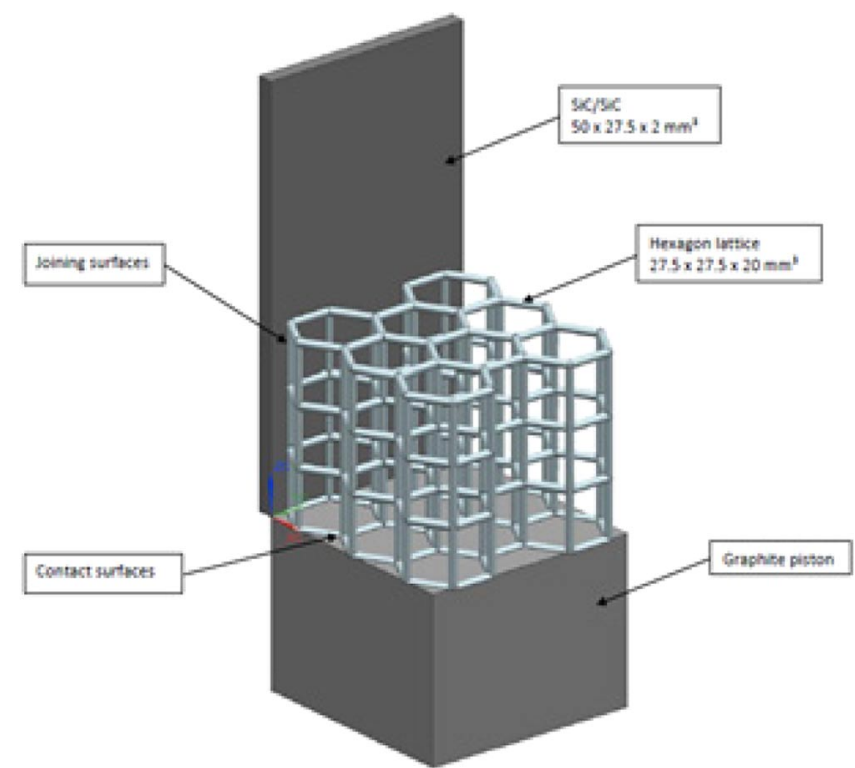

Fig. 9 Test setup for final joint characterization.

The basic performance of the joining techniques and subsequent optimization of the process is determined by pulloff tests and microstructural analysis. Base materials and joined specimens for the pulloff tests are shown in Fig. $\underline{8}$. Final selection among the three candidate routes will be based on the results of specific mechanical tests on shear strength at high temperature in the laboratories of Aerospace and Advanced Composites, GmbH.

A nonstandard procedure has been designed to properly test the reliability of the joint. A three-dimensional (3-D) sketch of the test setup is shown in Fig. 9. It includes a rectangular $(50 \times 27.5 \mathrm{~mm})$ sample representing the $\mathrm{CMC}$ skin. The samples are prepared by Erbicol from $\mathrm{SiC} / \mathrm{SiC}$ material with a thickness of $2 \mathrm{~mm}$ (see Fig. 8a). The lattice (see Fig. $\underline{\text { b }}$ ), consisting of three layers of eight hexagonal cells, is connected at the bottom of the sample. The cell size is identical to the cells that are going to be used for the L3K test hardware. Accordingly, the results from joint characterization can immediately be transferred to the test hardware.

During the tests, the external surface of the $\mathrm{SiC} / \mathrm{SiC}$ sample is being heated by radiation. In parallel, a mechanical load is applied by a graphite piston to the bottom side of the lattice, which is introducing a shear load to the joint. Two results will be obtained from the test, that is, 1) the upper temperature limit of the particular joint, and 2) the shear strength at the design temperature level. The design temperature level will be determined from the thermal tests in L3K on concept 0 . It is supposed to be below $1573 \mathrm{~K}$, which is considered as the maximal temperature.

Additional characterization tests are foreseen to determine the properties of the new CMC materials developed in the frame of the project, that is, 1) the $\mathrm{SiC} / \mathrm{SiC}$ material for the CMC skin of concept $2 \mathrm{a}$, and 2 ) the $\mathrm{C} / \mathrm{SiC}$ material with integrated highly conductive fibers used for the CMC skin of concept 1a. The tests include mechanical properties (tensile, compression, interlaminar shear strength, and

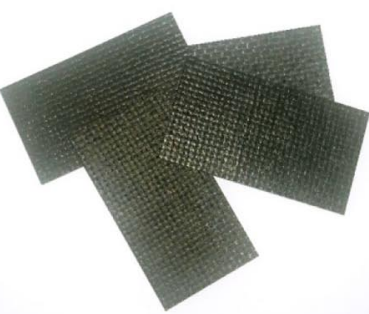

a) $\mathrm{SiC} / \mathrm{SiC}$ skin sample

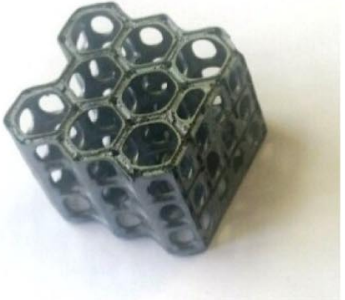

b) $\mathrm{Si}$-SiC lattice

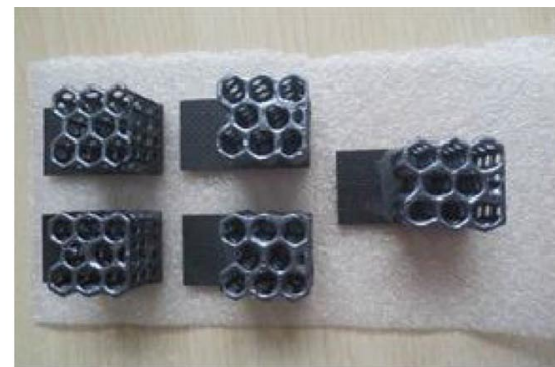

c) $\mathrm{CMC} /$ lattice assembly

Fig. 8 Base materials and specimen for joint characterization. 


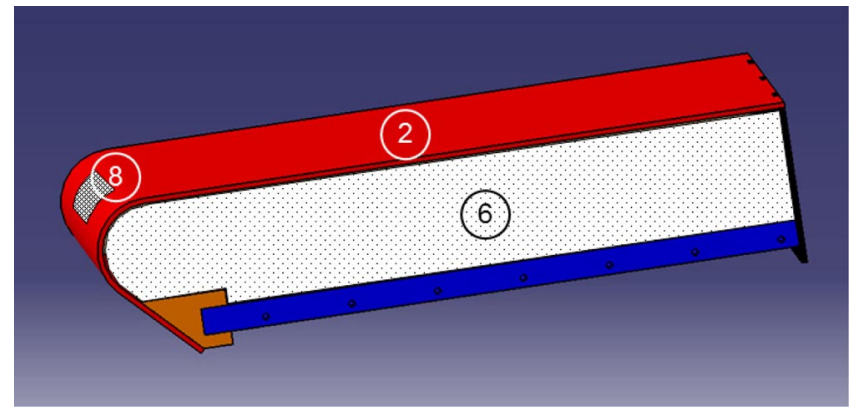

Fig. 10 Test configuration for concept $2 b$.

17

bending) according to proper standards (ASTM and EN) and thermophysical properties (thermal diffusivity, specific heat) at room temperature and at elevated temperature levels.

The second active cooling concept (i.e., concept $2 b$ ) is aiming at the transpiration of a coolant through the CMC skin, to locally decrease the surface temperature. The coolant is penetrating a porous segment, which is integrated into the TPS structure, toward the exterior. As shown in Fig. 10, an insert made of porous C/C 8 will be incorporated into the curved area of the CMC skin (2) to provide a permeable passage for gaseous coolants. The system requires a coolant supply system with a coolant reservoir attached to the porous segment.

It has been mentioned before that transpiration cooling is associated with the risk of significant boundary-layer interference. The interference with the boundary layer cannot be investigated in combination with the thermal validation of the concept in an archeated facility, but must be investigated at realistic and flight relevant Mach and Reynolds numbers. In the frame of THOR, a 18 corresponding test campaign is foreseen in Japan Aerospace Exploration Agency's high-enthalpy shock tunnel HIEST. A geometrically simple test setup is favorable for such tests. A planar configuration is much better suited than a curved one. Accordingly, the tests in HIEST are using a flat plate, as shown in Fig. 11.

In the upstream part, there is a removable insert, which can be equipped with a porous segment for transpiration cooling. The porous segment can be inserted flush bound into the flat plate. Coolant is supplied from a manifold below the flat plate. Measurements are foreseen in the wake of the porous segments. Heat flux and pressure measurements are performed by coaxial thermocouples and pressure transducers installed along the centerline of the flat plate. In particular, the heat flux distribution is providing information about boundary transition. An extension plate with additional sensors (pressure transducers and thermocouples) is attached to the front plate to obtain data up to a location nearly $1000 \mathrm{~mm}$ downstream of the porous segment.

The interaction between external flow and emanating coolant is visualized by high-speed schlieren photography. Tests are performed at several test conditions with different enthalpy, from low enthalpy $(3.5 \mathrm{MJ} / \mathrm{kg})$ to high enthalpy $(15 \mathrm{MJ} / \mathrm{kg})$. Different gaseous coolants are going to be applied with the flow rate as additional test parameters. In addition to nitrogen, which is considered as the baseline coolant, helium is applied.

\section{Experimental Verification}

Thermal validation tests are designated to be carried out in DLR, German Aerospace Research Center's arc-heated facility L3K. L3K is 1 one of the two test legs of the LBK facility, which has been playing an important role for decades in the qualification and testing of TPS components and materials [13]. Particular experience has been established in validation of fluid-structure interaction phenomena [9]. The test environment at L3K is particularly prepared for thermal measurements. Included are temperature measurements at specific locations in the interior of a model with several types of thermocouples, as well as surface temperature measurements. Pyrometers are used for local measurements, and in addition, surface temperature distributions on a complete model surface can be recorded with infrared camera systems.

Test conditions were selected from the set of the facility's standard operating conditions. One main criterion was applied for the selection: The test conditions should allow to performing of tests on the reference concept 0 , made of conventional passively cooled $\mathrm{CMC}$, without exceeding the material's operational limit temperatures. Two conditions, named THOR-1 and THOR-2, were considered. Both are associated with a gas mass flow rate of $142 \mathrm{~g} / \mathrm{s}$; the total enthalpy is $11.7 \mathrm{MJ} / \mathrm{kg}$ for THOR-1 and $11.0 \mathrm{MJ} / \mathrm{kg}$ for THOR-2. Based on a preparatory numerical study [14], condition THOR-2 was selected as the baseline test condition. For variation of the heat load gradient, the configurations are tested at two angles of attack: 10 and $20 \mathrm{deg}$. Although the maximal heat load at the stagnation line is supposed to be nearly identical for both angles, the average heat load on the model's top surface is lower for an inclination of $10 \mathrm{deg}$. Therefore, this configuration is associated with a higher load gradient [14].

The first experimental campaigns were carried out for the reference concept 0 and the two passive cooling concepts $1 \mathrm{a}$ and $1 \mathrm{~b}$. Test assemblies were prepared with the baseline geometry having a nose radius of $20 \mathrm{~mm}$. Photographs of the test assembly are given in Fig. 12. The CMC leading edge had been prepared with grooves for thermocouple integrated. In total, 15 thermocouples had been installed, eight of them along the symmetry line and three on each side in a distance of $60 \mathrm{~mm}$ to the symmetry line. Surface temperatures were measured with pyrometers at four spots along the symmetry line, each spot just above a thermocouple location. In addition, surface temperature distribution was recorded by an infrared camera system.

The test assembly for the reference concept was prepared with conventional CMC parts. For the tests related to concept 1a, the conventional CMC leading edge was replaced by another leading edge with integrated highly conductive fibers. With a value of $220 \mathrm{~W} / \mathrm{mK}$, the fibers' thermal conductivity is about one-order-ofmagnitude higher than the conductivity of the conventional fibers (see Fig. 2 to check the expected values for the resulting composites)

As shown in Fig. 13a, the interior of the model was completely filled with insulation material for the tests related to the reference concept and to concept $1 \mathrm{a}$. For concept $1 \mathrm{~b}$, the internal setup was changed to the configuration shown in Fig. 13b, integrated with conventional CMC, with a cavity opened behind the leading edge, which provides visibility for the radiative heat exchange. Each configuration was tested at test condition THOR-2 with different angles of attack: 10 and $20 \mathrm{deg}$.

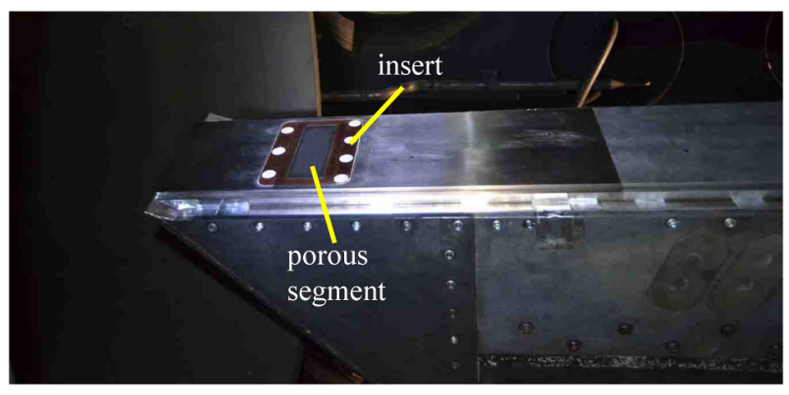

a) HIEST flat plate

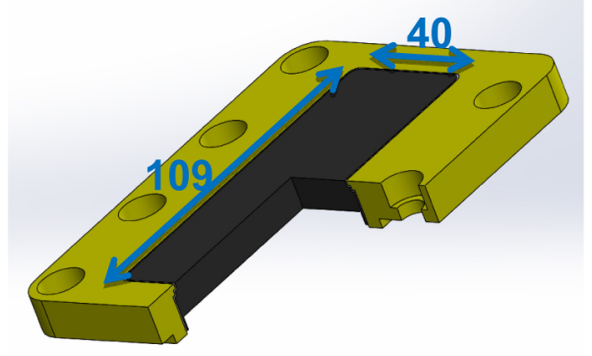

b) Test setup for the transpiration cooling

Fig. 11 Test setup for the transpiration cooling campaign in HIEST. 


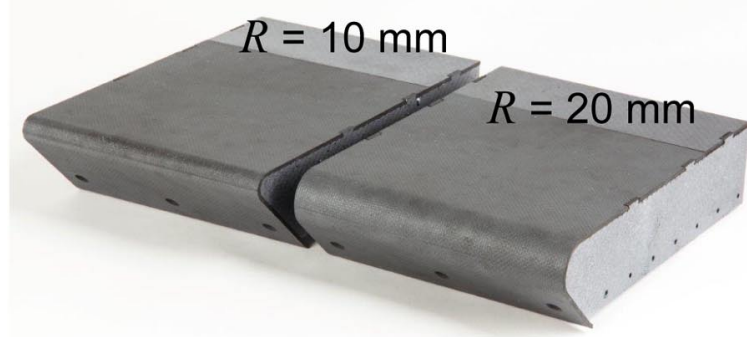

a) CMC Hardware

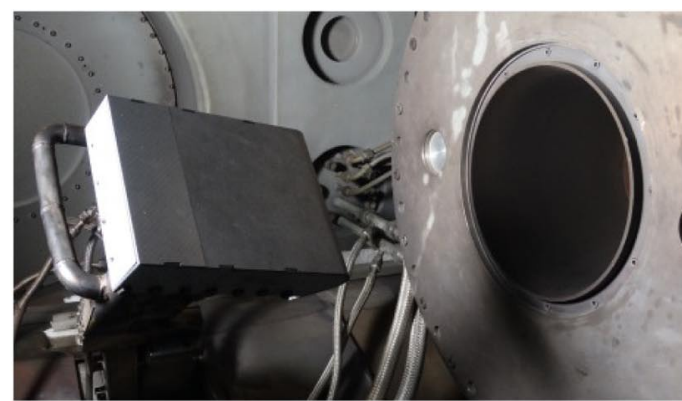

b) Test assembly in the L3K test chamber

Fig. 12 Experimental setup.

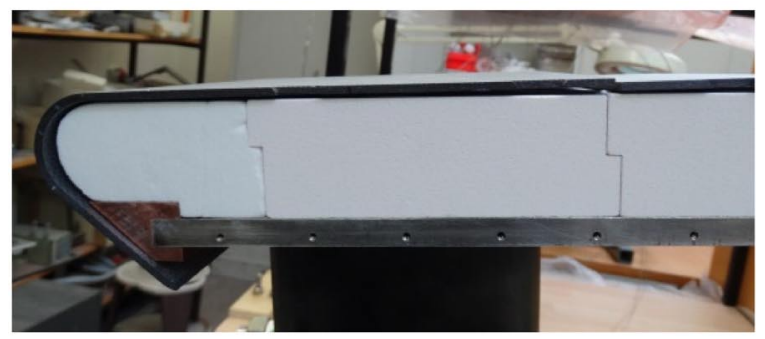

a) Reference concept and concept $1 \mathrm{a}$

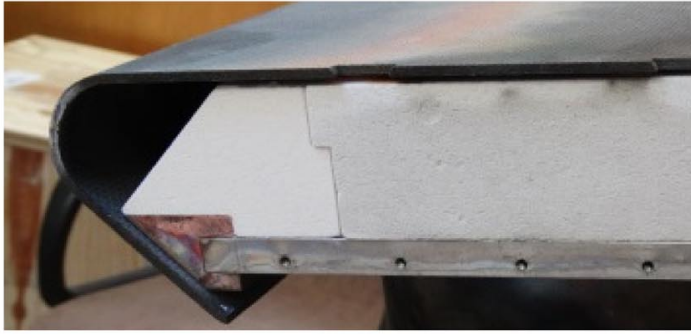

b) Concept $1 \mathrm{~b}$ (passive version)

Fig. 13 Model interior.

The test duration was $180 \mathrm{~s}$, which is sufficient to achieve steadystate conditions on the surface of the leading edge. Repeatability tests were included for each configuration to check the reproducibility of measured data.

Figure 14 shows results of three thermocouple measurements in the nose region, comparing the reference configuration with concept $1 \mathrm{~b}$ at a model inclination of $10 \mathrm{deg}$ (using conventional CMC).
All three thermocouples are placed on the symmetry line; the streamwise location is indicated in Fig. 14d.

When opening the cavity for concept $\overline{1 b}$, thermocouple (TC) 1 , on the backside of the stagnation point, and TC3, below the upper surface, are immediately affected, whereas TC4 remains covered by insulation material. With the open cavity, the temperature indicated by $\mathrm{TC} 1$ drops by $95 \mathrm{~K}$, whereas TC 3 indicates a temperature increase

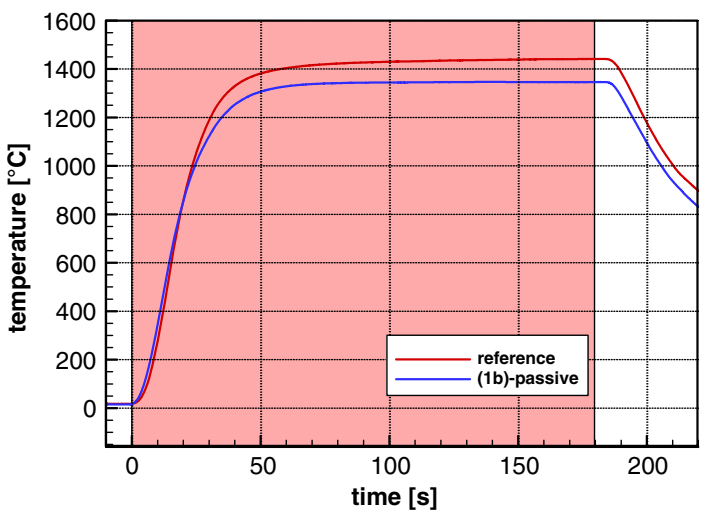

a) $\mathrm{TC1}$ recordings

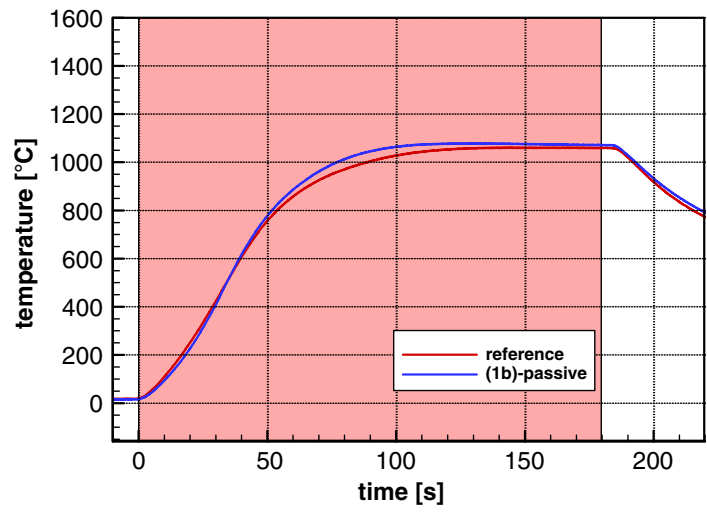

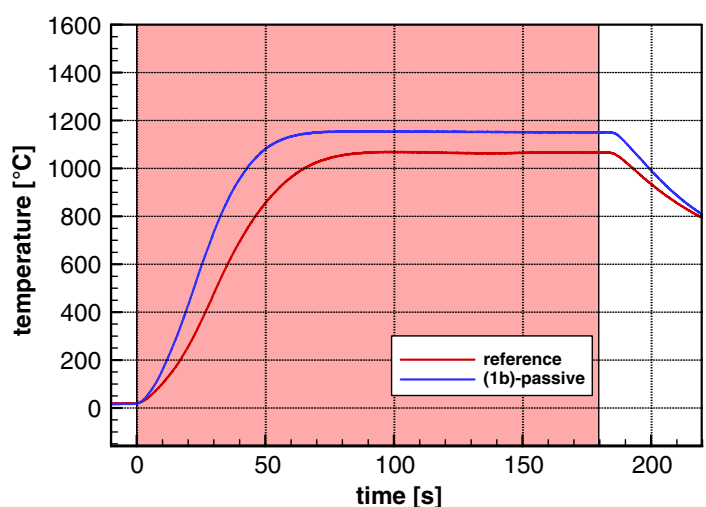

b) TC3 recording

TC $1 \quad$ TC $3 \quad$ TC 4

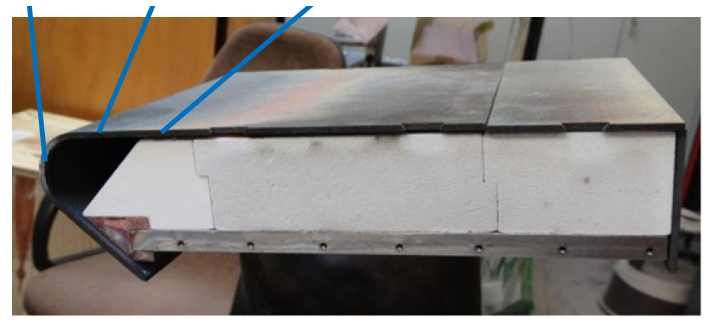

d) Thermo-couple locations

c) $\mathrm{TC} 4$ recording

Fig. 14 Cavity effect on thermocouple measurements (conventional CMC, $\alpha=10 \mathrm{deg}$ ). 


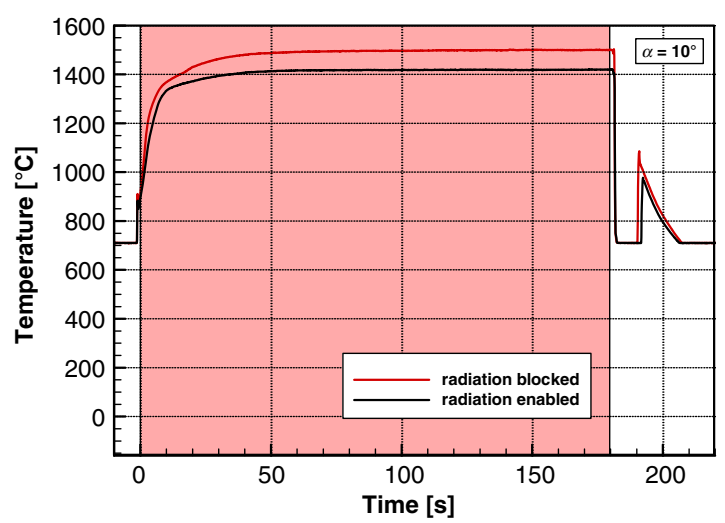

a) Conventional CMC

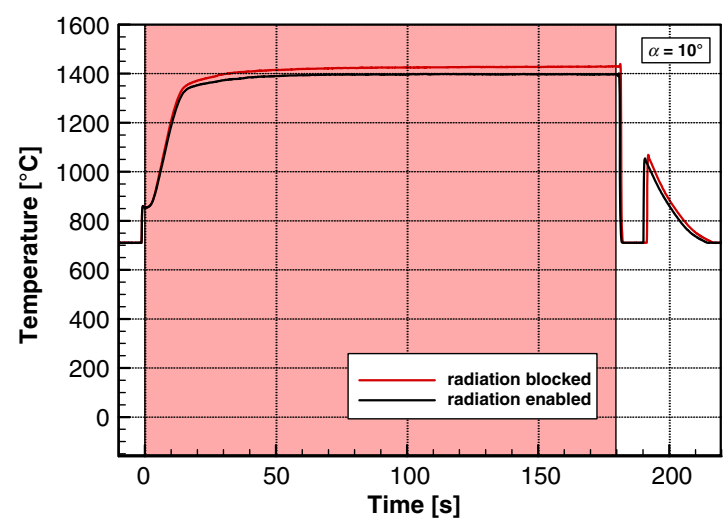

b) Highly conductive $\mathrm{CMC}$

Fig. 15 Cavity effect on stagnation temperature measurements (CMC, $\alpha=10 \mathrm{deg}$ ).

of $85 \mathrm{~K}$. And so, the temperature difference between TC1 and TC3, which amounts to $376 \mathrm{~K}$ in the reference configuration, is reduced to $194 \mathrm{~K}$ with the open cavity, clearly demonstrating a positive effect of thermal equilibration for this passive configuration. Thermocouple TC4 is hardly affected by the cavity. The difference between the two configurations is $14 \mathrm{~K}$.

A comparison of the surface temperature measurements at the stagnation point is plotted in Fig. 15, again for a model inclination of $10 \mathrm{deg}$. Compared with the reference, the passive version of concept $1 \mathrm{~b}$ provides a temperature reduction from 1500 to $1420^{\circ} \mathrm{C}$. This difference is considerable in terms of heat fluxes. According to the temperature reduction, the cavity effect enables the surface to sustain higher heat fluxes. With a reduction of $80 \mathrm{~K}$, the heat flux might be increased by $17 \%$.

The same comparison is shown in Fig. $15 \mathrm{~b}$ for the leading edge prepared with highly conductive CMC. Here, a temperature of $1430^{\circ}$ $\mathrm{C}$ was measured at the stagnation point with radiation blocked. This case refers to concept 1a. Accordingly, concept 1a provides a temperature reduction of $70 \mathrm{~K}$, allowing for an increase of heat flux by $15 \%$. When opening the cavity additionally, stagnation point temperature further reduces to $1397^{\circ} \mathrm{C}$. Accordingly, the combined application of concepts $1 \mathrm{a}$ and $1 \mathrm{~b}$ allows for an increase of the heat flux at the stagnation point, which is $21 \%$ above the heat flux at radiative equilibrium.

\section{Numerical Verification}

In addition to the experimental verification in high-enthalpy facilities, the four thermal concepts are going be verified numerically by coupled simulations related to the test configurations. Wellestablished simulation tools are being applied for flow and structure. Two flow solvers (i.e., DLR German Aerospace Research Center's
TAU [15] and Fluid Gravity Engineering's TINA codes) are available for the simulation of the high-enthalpy flows in the two facilities. Both are validated Navier-Stokes solvers, being capable of taking into account chemical as well as thermal nonequilibrium states for the fluid. During the project's conceptual phase, the TAU code had been applied in a preparatory study to identify the best suited operating conditions in $\mathrm{L} 3 \mathrm{~K}$, as described in the preceding section. The simulation of the thermomechanical response of the structure is carried out with commercial codes (e.g., ANSYS and MSC PATRAN).

Another preparatory numerical analysis was performed to determine the dimensional character of the leading-edge test configurations. In particular, the validity of the assumption, that a two-dimensional (2-D) analysis is sufficient, should be checked. In this context, 2- and 3-D computations of the flowfield around the leading-edge model were carried out. For simplification, a radiative equilibrium boundary condition was set on the model's surface. Some results are plotted in Fig. 16. Figure 16a shows some temperature isolines on the model surface. Starting at the symmetry plane on the left, the isolines run in the lateral direction mainly.

Deviations from this trend are observed in close vicinity to the model's side edge only, indicating a mainly two-dimensional character. This hypothesis is confirmed by Fig. 16b, in which surface temperature distributions in several cutting planes are plotted and compared with the results of a 2-D simulation. Except for the cut at $y=0.09 \mathrm{~m}$ (i.e., in $7 \mathrm{~mm}$ distance to the sidewall), all curves appear coincident with the 2-D result.

For coupled simulations, a loosely coupled strategy is applied. Starting from an initial distribution of convective heat fluxes (e.g., based on radiative equilibrium at the surface), the resulting surface temperatures are computed by the structural code. This result is transferred to the flow solver to correct the convective heat fluxes for

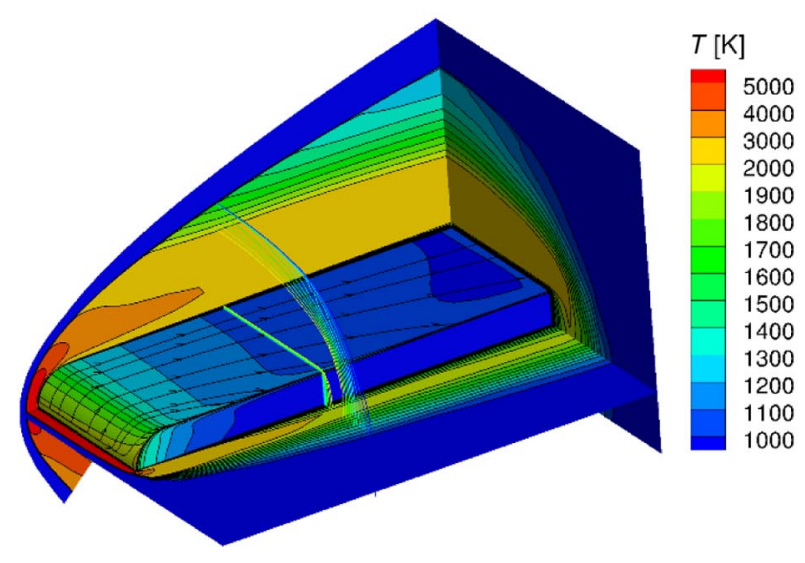

a) 3D view of temperature isolines and streamlines

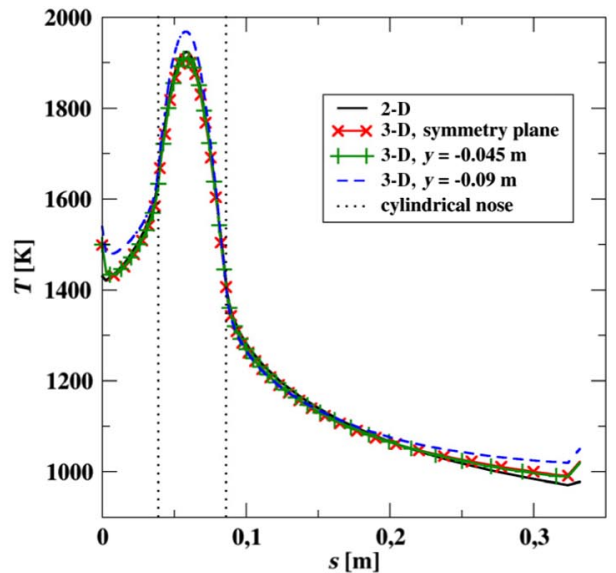

b) Surface temperatures distributions

Fig. 16 Numerical results of 3-D flow simulation assuming radiative equilibrium at model surface. 


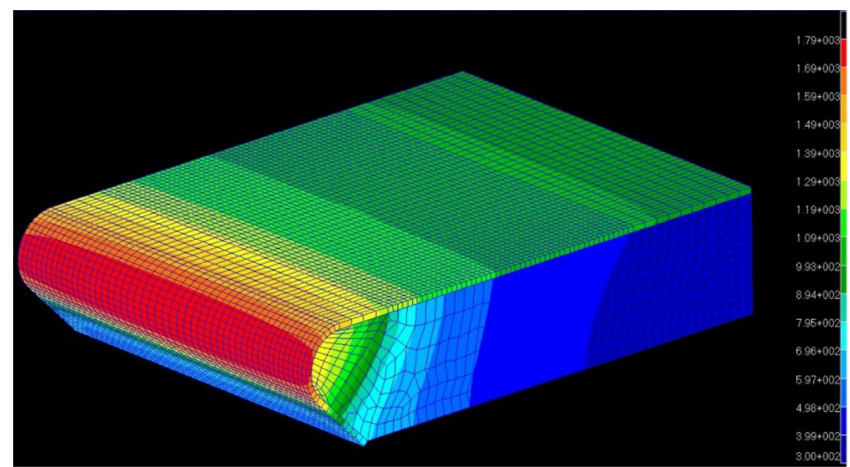

Fig. 17 Surface temperature distribution for passive version of concept $1 \mathrm{~b}$ (result from a coupled simulation).

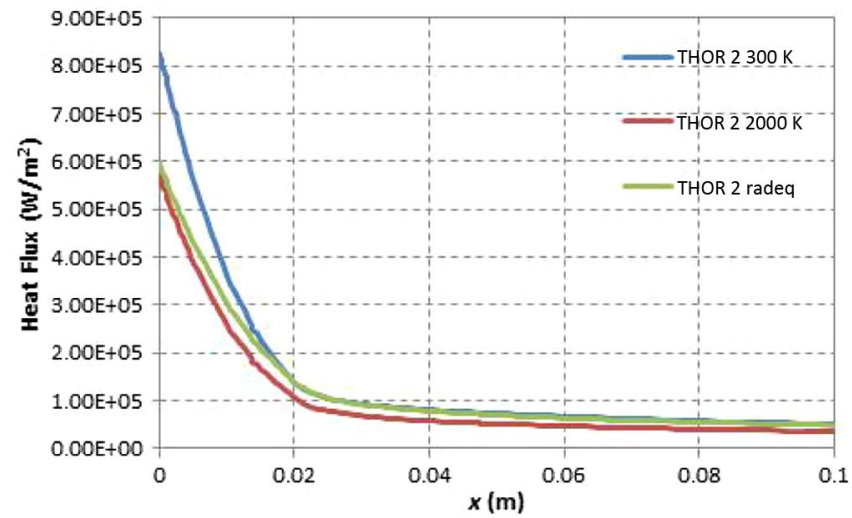

Fig. 18 Surface noncatalytic heat flux distributions at test condition THOR-2.

the next iteration. Generally, the procedure converges within a few iterations. As an example, an intermediate result of the surface temperature distribution is shown in Fig. 17. The plot refers to the thermal response of the leading-edge model for the passive version of concept $1 \mathrm{~b}$ (see Figs. 12a and 13b).

A slightly different approach is followed for the numerical simulations related to the active cooling concept 2a. This configuration is the most complex one. In addition to the external flow, it includes a complex internal flow that heavily interacts with the CMC skin and the ceramic lattice. For the coupling with the external flow, an aerothermodynamic database is used. The database can be prepared in advance and provides the link between surface temperature and heat flux rate. The data were generated from several simulations with the TINA code. Heat fluxes and surface temperatures were determined for noncatalytic and supercatalytic surface conditions at several L3K flow conditions and for various model inclinations. For the noncatalytic case, Fig. 18 exemplarily shows the heat flux distribution for radiative equilibrium conditions at test condition THOR-2, compared with the results of two isothermal wall conditions.

For concept $2 \mathrm{a}$, detailed simulations of the flow inside the CMC sandwich structure were carried out to identify the optimal flowpath for the coolant and to check the performance of this concept. Radiative equilibrium conditions were used for the thermal load on the external surface. For the baseline test configuration, three different flowpaths were considered. The results were compared with regard to the overall temperature level, the lateral homogeneity of the surface temperatures, and the prevention of a recirculation zone within the lattice. Best results were obtained for a flowpath following the contour of the leading edge, with an inlet at the bottom and outlet at the top surface, as sketched in Fig. 19.

The surface temperature shows a homogeneous distribution in the lateral direction with only minor variations close to the sides. Using air as coolant, with a flow rate of $2.5 \mathrm{~g} / \mathrm{s}$, a maximal surface temperature of $1630 \mathrm{~K}$ is achieved, which is considerably below the radiative equilibrium temperature of about $1900 \mathrm{~K}$ (see Fig. 19b).

\section{Conclusions}

The FP7 project THOR is targeting new thermal management concepts for atmospheric space entry and hypersonic transport vehicles. A leading edge has been selected as the reference configuration and corresponding requirements were collected. Four different concepts (two passive and two active) are being traded off, by simulation and testing, to obtain an assured solution after a verification campaign.

The first passive concept 1a, using a CMC containing highly conductive pitch-based carbon fibers, shows conclusive evidence of improved thermal equilibration, both numerically and experimentally. The second passive concept $1 \mathrm{~b}$ uses cavities to enhance balancing of thermal loads by internal radiation. Experimental results indicate a significant potential for thermal equilibration. The first active concept 2 a envisages the use of ceramic porous structures to drive and promote heat exchange with a coolant gas by means of convective cooling. Sound joining with CMC walls is a key issue. Numerical simulation is quite encouraging; joining and assembly activities are ongoing. The second active concept $2 \mathrm{~b}$ is addressed to transpiration cooling, where a porous $\mathrm{C} / \mathrm{C}$ insert will be integrated into the test configuration for experimental verification in L3K (DLR, German Aerospace Research Center) and the HIEST shock tunnel (Japan Aerospace Exploration Agency, JAXA). Preliminary tests at DLR demonstrated feasibility of the concept and preliminary tests at JAXA were conducted successfully.

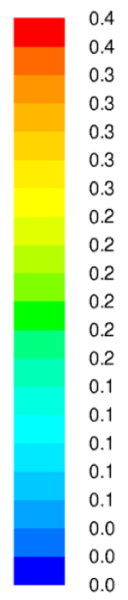

a) Surface temperature
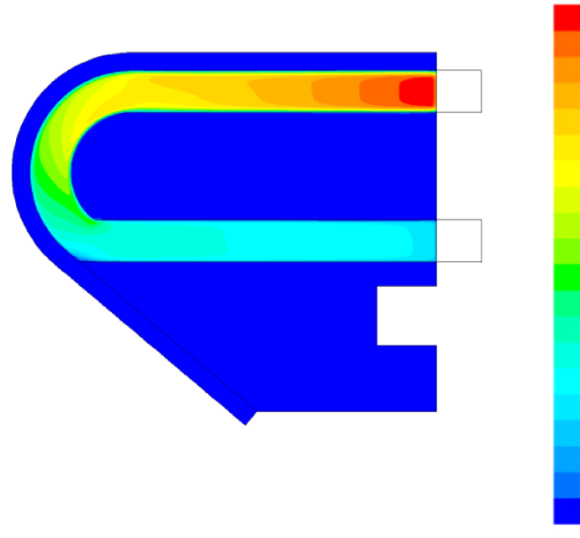

b) Mach number (internal flow only)

Fig. 19 Numerical results for concept 2a (coolant: air at $2.5 \mathrm{~g} / \mathrm{s}$ ). 


\section{Acknowledgments}

The research leading to these results has received funding from the European Union Seventh Framework Programme (FP7/2007-2013) under grant agreement 312807 . The authors are thankful for technical support from Cristina Jimenez and Xabier Hernandez (Tecnalia); Ivaylo Petkov, Volker Hannemann, and Oliver Hohn (DLR, German Aerospace Center); Jamie Caldwell and Jim Merryfield (Fluid Gravity Engineering, Ltd.); Suat Ontac (Tubitak Uzay Space Technology Research Institute); Luca Ferrari and Maurizio Barbato (Scuola Universitaria Professionale della Svizzera Italiana); and Mauricio Portaluppi, Daniela Santella, and Angelo Stio (Thales Alenia Space).

\section{References}

[1] Esser, B., Gülhan, A., Kuhn, M., Petkov, I., Hannemann, V., Barcena, J., Jimenez, C., Okan, A., Ontac, S., Merrifield, J., Gianella, S., Gaia, D., Ortona, A., Barbato, M., Liedtke, V., Francesconi, D., Portaluppi, M., Santella, D., and Hideyuki, T., "Innovative Thermal Management Concepts for Sharp Leading Edges of Hypersonic Vehicles," Materials Science and Technology Conference, MS\&T' 14, Oct. 2014.

[2] Serre, L., "Overview of Hypersonics in Europe," 18th AIAA International Space Planes and Hypersonic Systems and Technology Conference, AIAA, Reston, VA, Sept. 2012.

[3] Longstaff, R., "From HOTOL to SKYLON. British Spaceplane Programs: Past, Present and Future," 18th AIAA International Space Planes and Hypersonic Systems and Technology Conference, AIAA, Reston, VA, Sept. 2012.

[4] Sippel, M., Schwanekamp, T., Bauer, C., Garbers, N., van Foreest, A., Tengzelius, U., and Lentsch, A., "Technical Maturation of the SpaceLiner Concept," 18th AIAA International Space Planes and Hypersonic Systems and Technology Conference, AIAA, Reston, VA, Sept. 2012, pp. 14-18.

[5] Steelant, J., and Langener, T., "The LAPCAT-MR2 Hypersonic Cruiser Concept," 29th Congress of the International Council of the Aeronautical Sciences, Sept. 2014.

[6] Bouchez, M., Crampon, F., Le Naour, B., Wilhelmi, C., Bubenheim, K., Kuhn, M., Mainzer, B., Riccius, J., Davoine, C., Justin, J.-F., von Wolfersdorf, J., Abdelmoula, M., Villace, V. F., and Steelant, J., "Combustor and Material Integration for High Speed Aircraft in the European Research Program ATLLAS2," 19th AIAA Aviation and Aeronautics Forum and Exposition, American Institute of Aeronautics and Astronautics, AIAA, Reston, VA, June 2014.
[7] Detra, R. W., Kemp, N. H., and Riddell, F. R., "Addendum to 'Heat Transfer to Satellite Vehicles Re-Entering the Atmosphere'," Jet Propulsion, Vol. 27, 1957, pp. 1256-1257.

[8] Reimer, T., Petkov, I., and Rotärmel, W., "Numerical Investigation of the Thermal Benefit of Using a High Thermal Conductivity CMC for the Leading Edge of Hypersonic Vehicles," Proceedings of the Eighth European Symposium on Aerothermodynamics of Space Vehicles, ESA Publ., Noordwijk, The Netherlands, 2015.

[9] Willems, S., Esser, B., and Gülhan, A., "Experimental and Numerical Investigation on Thermal Fluid-Structure Interaction on Ceramic Plates in High Enthalpy Flow," CEAS Space Journal, Vol. 7, No. 4, 2015, pp. 483-497. doi: 10.1007/s12567-015-0101-5

[10] Ortona, A., Pusterla, S., and Gianella, S., "An Integrated Assembly Method of Sandwich Structured Ceramic Matrix Composites," Journal of the European Ceramic Society, Vol. 31, No. 9, 2015, pp. 1821-1826. doi:10.1016/j.jeurceramsoc.2011.03.010

[11] Kuhn, M., and Hald, H., "Application of Transpiration Cooling for Hot Structures," RESPACE - Key Technologies for Reusable Space Systems, Notes on Numerical Fluid Mechanics and Multidisciplinary Design, edited by Gülhan, A., Springer-Verlag, Berlin, 2008, pp. 82103. doi:10.1007/978-3-540-77819-6

[12] Reimer, T., Esser, B., and Gülhan, A., "Arc Jet Testing of CMC Samples with Transpiration Cooling," 44th AIAA Thermophysics Conference, AIAA, Reston, VA, 2013, pp. 2013-2904.

[13] Gülhan, A., and Esser, B., "Arc-Heated Facilities as a Tool to Study Aerothermodynamic Problems of Reentry Vehicles," Advanced Hypersonic Test Facilities, edited by Lu, F. K., and Marren, D. E., Vol. 198, Progress in Astronautics and Aeronautics, AIAA, Reston, VA, 2002, pp. 375-403.

[14] Esser, B., Gülhan, A., Reimer, T., and Petkov, I., "Experimental Verification of Thermal Management Concepts for Space Vehicles," Proceedings of the Eighth European Symposium on Aerothermodynamics of Space Vehicles, ESA Publ., Noordwijk, The Netherlands, 2015.

[15] Schwamborn, D., Gerhold, T., and Heinrich, R., "The DLR TAU-Code: Recent Applications in Research and Industry," European Conference on Computational Fluid Dynamics, ECCOMAS CFD 2006, edited by Wesseling, P., Onate, E., and Periaux, J., Delft Univ. of Technology, Delft, The Netherlands, 2006

Dr. T. K. Minton Associate Editor 


\section{Queries}

1. AU: Please spell out Str. in the footnote instead of using the abbreviation.

2. AU: Please check that the copyright (@) type is correct. Note that the code will be added upon publication.

3. AU: Please check that the affiliations and affiliation footnotes are correct; divisions, departments, and institutes are given in the footnotes.

4. AU: Minor syntax adjustments were made throughout; please read closely to confirm that your meaning was retained.

5. AU: Acronyms have been removed from the Abstract per journal style. They are defined in the main text.

6. AU: If THOR is an acronym, please spell out in the Abstract and remove the acronym, then define THOR the first time it is used in the main text.

7. AU: Acronyms have been removed from the Abstract per journal style. They are defined in the main text.

8. AU: The subscript for hot wall was changed from $\mathrm{cw}$ to hw in the nomenclature. Please confirm this is correct.

9. AU: Instructions from AIAA indicate that figures are to appear in color only online. Please verify the usage of color in your proof is correct, and note that all figures will be grayscale in the print journal.

10. AU: Lists that do not consist of complete sentences are run in the with the text. Please ensure your meaning has been retained.

11. AU: Subsequent uses of quotation marks around the same term have been removed per journal style.

12. AU: Acronyms not used again in the paper have been removed per journal style.

13. AU: Please confirm the definition added for SUPSI is correct or redefine.

14. AU: Please spell out the chemical Si-SiC so that the caption of Fig. 7 does not begin with an abbreviation.

15. AU: Please confirm the definition for AAC is correct or redefine.

16. AU: If $\mathrm{L} 3 \mathrm{~K}$ is an acronym/abbreviation, please define the first time used in the text.

17. AU: Please define ASTM and EN and remove the acronyms since they are not used again in the paper, per journal style.

18. AU: If HIEST is an acronym, please define at first use in the main text.

19. AU: Please define LBK and remove the acronym.

20. AU: The sentence "The CMC leading edge had been prepared with grooves for thermocouple integrated" is not a complete sentence and is unclear. Please review and revise as needed.

21. AU: "Thermocouple" was added as the definition for TC in the text. Please confirm this is correct, or redefine. 
22. AU: Please confirm the definition added for FGE is correct or redefine.

23. AU: Acronyms are not used in the Acknowledgments. Please confirm the definitions used in place of the acronyms are correct.

24. AU: If Refs. [1-3, 5, 6] are published proceedings, please provide the names and locations of the publishers (not to be confused with the conference host and location) and the page range. If they are conference papers, please provide the paper numbers and the organizers' names. If they are CD-ROMs, please provide the names and locations of the CD-ROM producers.

25. AU: Please provide the issue number for [7].

26. AU: Please provide a page range for information used in $[8,14,15]$. 